\title{
Disparity in fasting plasma cholesterol and abdominal adiposity within and between genders in Lagos Southwest Nigeria
}

\begin{abstract}
Objective: Though the biochemical structure of cholesterol, high density lipoprotein and low density lipoprotein are the same in both gender, yet their distribution differs. These observed differences provoke the question how many male and female with high abdominal adiposity have high plasma cholesterol level (hyperlipidemia)? This paper aim to study this disparity in the lipidology of both sexes in Southwest Nigeria, among Lagosian.
\end{abstract}

Methods: These data were obtained during a free health screening services in surulere area of Lagos. The fasting total cholesterol level was taken by the use of a cholesterol meter, a fasting cholesterol of $200 \mathrm{mg} / \mathrm{dl}$ or less was taken as normal. In this study, abdominal adiposity was assessed clinically with the use of a non stretch tape to measure waist circumference at the level of the umbilicus. A waist circumference of $94 \mathrm{~cm}$ and above and greater than or equal to $80 \mathrm{~cm}$ were taken as high abdominal adiposity (central obesity) for male and female respectively.

Results: A total of 22 males and 49 females were involved in the study making a total of 71 participants. The age range among male studied was $31-70$ years with a mean age of 49.45 years. Of these $72.72 \%$ (16) men had normal abdominal adiposity while $27.27 \%$ (6) had high abdominal adiposity. Also $18.18 \%$ (4) had hyperlipidaemia, only $25 \% 1$ of those with hyperlipidaemia had high abdominal adiposity while the remaining $75 \%$ had normal abdominal adiposity despite being hyperlipidemic. Similarly among those (6) with high abdominal adiposity, only $16.67 \%$ (1) had hyperlipidemia, in the female gender, the age range was 18 -88years with a mean age of 46.29 years. Of these $20.41 \%$ (10) had normal abdominal adiposity while $79.59 \%$ (39) had high abdominal adiposity. Also $28.57 \%$ (14) had hyperlipidemia. Of the female subjects with hyperlipidaemia, $78.57 \%$ (11) had high abdominal adiposity while $21.43 \%$ (3) had normal abdominal adiposity. However, among those with high abdominal adiposity in the female gender, only $28.21 \%$ (11) had hyperlipidemia.

Discussion: In both gender, it is not all that have high abdominal adiposity that have hyperlipidemia and it is not all that have hyperlipidemia that have high abdominal adiposity. A marked disparity was noticed between gender with respect to those with hyperlipidaemia and high abdominal adiposity. In male subjects with hyperlipidaemia the subject with high abdominal adiposity is in the minority $(25 \%)$ while in female subjects with hyperlipidemia, those with high abdominal adiposity are in the majority $(78.57 \%)$. The disparity here is marked and of significant prognostic importance.

Conclusion: In both gender minority number of those with high abdominal adiposity have hyperlipidaemia though with female having sizable minority number. Conversely a marked disparity is seen among female subjects with hyperlipidemia.

Keywords: hyperlipidemia, abdominal adiposity, gender, disparity
Volume 6 Issue 3 - 2018

Ademolu B Adegbenga

Department of Medicine, Lagos State University Teaching Hospital, Nigeria

Correspondence: Ademolu B Adegbenga, Department of Medicine, Lagos State University Teaching Hospital, Ikeja, Lagos, Nigeria,Email ademoluab@yahoo.com

Received: April II, 2018 | Published: June 22, 2018

\section{Introduction}

Though the numbers of chromosomes in both gender nuclei are the same, the phenotypic appearance of each gender are different. Though the biochemical structure of cholesterol, high density lipoprotein, low density lipoprotein and very low density lipoprotein are the same in both gender, yet their distribution and plasma levels differs. These observed differences provokes the following questions how many male with high abdominal adiposity have high cholesterol level (hyperlipidaemia)? How many male and female with high abdominal adiposity have normal total cholesterol level? How many male and female with normal abdominal adiposity have high plasma cholesterol level? It is well known that most female stores their fat in the hip while most male store theirs in the abdomen. This paper aim to study these disparities in the lipid logy of both sexes in southwest Nigeria, among Lagosian.

Different technique of measurement of abdominal adiposity varied with different degree of predictive value. While the use of non stretch tape to measure waist circumference and/or waist hip ratio both of which are clinical measurement of Abdominal Adiposity with different precision, is easy and universally acceptable as it is 
approved by the World Health Organisation, other relevant methods used in measurement of abdominal adiposity include computerised tomographic scan, magnetic resonant imaging , underwater weighing, isotope dilution, whole-body dual-energy x-ray absorptiometry (DEXA) and Bio-impedance analysis. ${ }^{1}$ It is noted that both Abdominal Adiposity and overall obesity strongly and independently predict the risk of type 2 diabetes mellitus. ${ }^{2}$

\section{Methodology}

These data were obtained during a free health screening services in Surulere area of Lagos. The participants were randomly selected after taking informed consent. The fasting total cholesterol level was taken by the use of a cholesterol meter, a fasting cholesterol of $200 \mathrm{mg} /$ $\mathrm{dl}$ or less was taken as normal. In this study, abdominal obesity was assessed clinically with the use of a non stretch tape to measure waist circumference at the level of the umbilicus at minimal expiration. A waist circumference of $94 \mathrm{~cm}$ and above and greater than or equal to $80 \mathrm{~cm}$ were taken as high abdominal adiposity (central obesity) for male and female respectively according to the European cut offs for waist circumference. A total of 22 males and 49 females were involved in the prospective study making a total of 71 participants. The limitation of the study is that waist circumference was used as a measurement of abdominal adiposity, the use of computerised tomography, or magnetic resonant imaging to estimate abdominal adiposity would have been a better objective assessment of abdominal adiposity but this is not economically feasible in this prospective study in a developing country like Nigeria. The exclusion criteria include pregnant women, ascites of any cause or anasarca. The data were analysed using SPSS version 17.0.

\section{Statistical analysis}

By using descriptive statistics, the mean of the ages was 47.3 years with a standard error of the mean of 1.79 . The median age was 49 years with a multiple mode of 31 and 52 years and a standard deviation of 15.11 with a skewness of 0.155 . The age range was 70 years. The mean of the cholesterol was $177 \mathrm{mg} / \mathrm{dl}$ with a standard error of the mean of 4.10. The median cholesterol value was $179 \mathrm{mg} / \mathrm{dl}$ with a mode of 140 $\mathrm{mg} / \mathrm{dl}$ and a standard deviation of 34.56 with a skewness of 0.710 . The range was $53 \mathrm{mg} / \mathrm{dl}$. The least cholesterol reading was $137 \mathrm{mg} / \mathrm{dl}$ while the highest value was $281 \mathrm{mg} / \mathrm{dl}$. In this study, the mean of the waist circumference was $88.95 \mathrm{~cm}$ with a standard error of the mean of 1.47 . The median waist circumference in the sample size was $89 \mathrm{~cm}$ with a mode of $86 \mathrm{~cm}$ and a standard deviation of 12.37 with a skewness of 0.35 . The range was $53 \mathrm{~cm}$. The lowest waist circumference was $65 \mathrm{~cm}$ while the largest was $118 \mathrm{~cm}$ (Table 1).

By using the pearson correlation, age correlated with waist circumference with value of 0.400 with $p$-value of 0.001 while it correlated to a lesser degree with cholesterol with value of 0.214 with a p-value of 0.074.As expected age did not correlate with gender. Cholesterol did not correlate significantly to gender and waist circumference with values of 0.076 and 0.065 respectively with p-values of 0.529 and 0.590 . The pearson correlation between waist circumference and gender was 0.115 with a p-value of 0.339 (Table 2).
Table I Descriptive Statistic of Disparity in Jasting Plasma Cholesterol and Abdominal Adiposity in Lagos Southwest Nigeria

\begin{tabular}{|c|c|c|c|c|}
\hline & Age & Gender & Cholesterol & WC \\
\hline \multirow[t]{2}{*}{$\mathbf{N}$} & 71 & 71 & 71 & 71 \\
\hline & 0 & 0 & 0 & 0 \\
\hline Mean & 47.2676 & 1.6901 & 177.0282 & 88.9507 \\
\hline Std. Error of Mean & 1.79424 & 0.05527 & 4.10109 & 1.46787 \\
\hline Median & 49 & 2 & 179 & 89 \\
\hline Mode & $31.00^{\mathrm{a}}$ & 2 & 140 & 86 \\
\hline Std. Deviation & 15.11854 & 0.46573 & 34.55636 & 12.3685 \\
\hline Variance & 228.57 & 0.217 & 1194.142 & 152.98 \\
\hline Skewness & 0.155 & -0.84 & 0.71 & 0.035 \\
\hline $\begin{array}{l}\text { Std. Error of } \\
\text { Skewness }\end{array}$ & 0.285 & 0.285 & 0.285 & 0.285 \\
\hline Range & 70 & 1 & 144 & 53 \\
\hline Minimum & 18 & I & 137 & 65 \\
\hline Maximum & 88 & 2 & 281 & 118 \\
\hline Sum & 3356 & 120 & 12569 & 6315.5 \\
\hline
\end{tabular}

aMultiple modes exist. The smallest value is shown.

Table 2 Correlation between Age, Gender, Plasma Cholesterol and Waist Circumference (WC) in Lagos, Southwest Nigeria

\begin{tabular}{|c|c|c|c|c|c|}
\hline & & Age & Gender & Cholesterol & Wc \\
\hline \multirow{3}{*}{ Age } & $\begin{array}{l}\text { Pearson } \\
\text { Correlation }\end{array}$ & 1 & -0.098 & 0.214 & $.400 * *$ \\
\hline & Sig. (2-tailed) & & 0.418 & 0.074 & 0.001 \\
\hline & $\mathrm{N}$ & 71 & 71 & 71 & 71 \\
\hline \multirow{3}{*}{ Gender } & $\begin{array}{l}\text { Pearson } \\
\text { Correlation }\end{array}$ & -0.098 & 1 & 0.076 & 0.115 \\
\hline & Sig. (2-tailed) & 0.418 & & 0.529 & 0.339 \\
\hline & $\mathrm{N}$ & 71 & 71 & 71 & 71 \\
\hline \multirow{3}{*}{ Cholesterol } & $\begin{array}{l}\text { Pearson } \\
\text { Correlation }\end{array}$ & 0.214 & 0.076 & 1 & 0.065 \\
\hline & Sig. (2-tailed) & 0.074 & 0.529 & & 0.59 \\
\hline & $\mathrm{N}$ & 71 & 71 & 71 & 71 \\
\hline \multirow{3}{*}{ WC } & $\begin{array}{l}\text { Pearson } \\
\text { Correlation }\end{array}$ & $.400^{\text {** }}$ & 0.115 & 0.065 & 1 \\
\hline & Sig. (2-tailed) & 0.001 & 0.339 & 0.59 & \\
\hline & $\mathrm{N}$ & 71 & 71 & 71 & 71 \\
\hline
\end{tabular}

**.Correlation is significant at the 0.0 l level (2-tailed).

\section{Results}

A total of 22 males and 49 females were studied. Hence the total population studied was 71 . The age range among male studied was $31-70$ years with a mean age of $49.45 y$ years. Of these men $72.72 \%$ (16) had normal abdominal adiposity while $27.27 \%$ (6) had high abdominal adiposity (Figure 1). Also $18.18 \%$ (4) had hyperlipidaemia 
and all are in their sixth decades of life, only $25 \%$ (1) of those with hyperlipidaemia had high abdominal adiposity while the remaining $75 \%$ had normal abdominal adiposity despite being hyperlipidaemic (Figure 2). Similarly among those (6) with high abdominal adiposity, only $16.67 \%$ (1) had hyperlipidaemia, the remaining $83.3 \%$ (5) had normal cholesterol level despite high abdominal adiposity (Figure 3 ).

In the female gender, the age range of the studied female population is $18-88$ years with a mean age of 46.29 years. Of these $20.41 \%$ (10) had normal abdominal adiposity while 79.59\% (39) had high abdominal adiposity (Figure 2). Also 28,57\% (14) had hyperlipidaemia while the remaining $71.43 \%$ had normal plasma lipid level (Figure 4).The distribution of hyperlipidaemia against decades of life in female shows none in the 2nd decade of life, $25 \%$ (2) in 3 rd decade of life, $50 \%$ (3) in 4th decade of life, $25 \%$ (3) in the 5 th decades of life, $21.43 \%$ (3) in the 6th decades of life, $60 \%$ (3) in the 7 th decades of life and none in the 8th and 9th decades of life in this study (Figure 5). Of the female subjects with hyperlipidaemia,78.57\% (11) had high abdominal adiposity while $21.43 \%$ (3) had normal abdominal adiposity (Figure 2). While female subjects with both hyperlipidaemia and high abdominal adiposity span through the 3rd to 7 th decade of life, the three female subjects with hyperlipidaemia and normal abdominal adiposity are one in 4th decades of life, and one each in 6th and 7th decades of life. However, among those with high abdominal adiposity in the female gender, only $28.21 \%$ (11) had hyperlipidaemia, the remaining $71.79 \%$ had normal fasting cholesterol level despite having high abdominal adiposity (Figure 3 ).

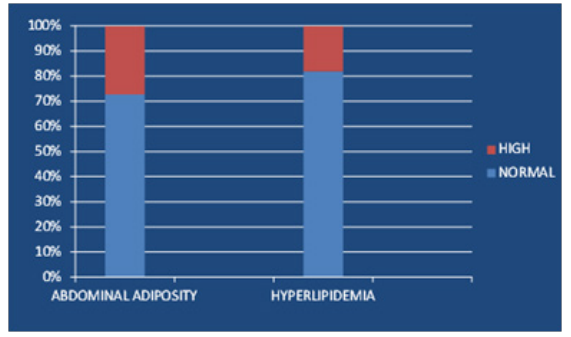

Figure I Distribution of Abdominal Adiposity and Hyperlipidemia in Male.

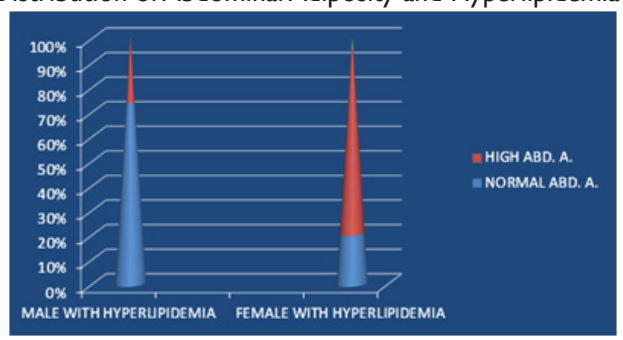

Figure 2 Marked Gender Disparity in Abdominal Adiposity with Baseline Hyperlipidemia.

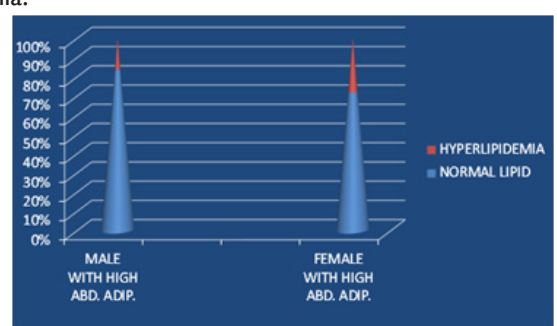

Figure 3 Distribution of Hyperlipidemia with Baseline high Abdominal Adiposity in both Genders.

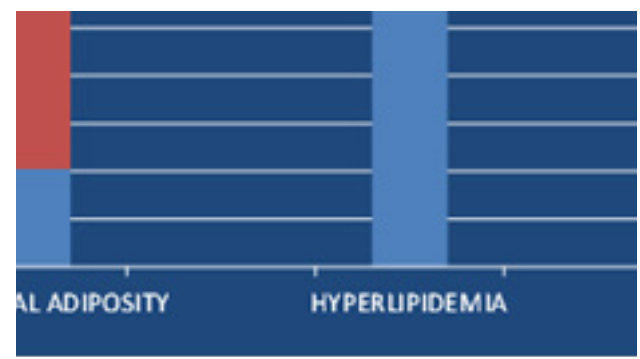

Figure 4 Distribution of Abdominal Adiposity and Hyperlipidemia in Female.

\section{Discussion}

Regional body fat distribution has an important influence on metabolic and cardiovascular risk factors. Many prospective studies have shown that increased abdominal (visceral) fat accumulation is an independent risk factor for Coronary Artery Disease, hypertension, stroke, and type 2 diabetes (DM2). ${ }^{3}$ The changes in lipid metabolism seen with abdominal fat accumulation have been well characterized and include hypertriglyceridemia, reduced HDL cholesterol, and increased numbers of small, dense LDL particles. Elevated LDL cholesterol is not a feature of the dyslipidemia seen with abdominal obesity. Other features of the dyslipidemia of abdominal adiposity include elevated very low density lipoproteins (VLDL), and reduced $\mathrm{HDL},{ }^{2}$ which are the large buoyant antiatherogenic subspecies of total HDL. In some individuals, apo B levels may be elevated, reflecting an increase in the number of small, dense lipoprotein particles (VLDL and LDL). ${ }^{3}$ An increased number of small, dense LDL particles is a constant feature of the dyslipidemia of abdominal adiposity, as they are associated with insulin resistance, intraabdominal fat, and hypertension. ${ }^{4-6}$ Even normal weight individuals with increased amounts of abdominal adipose tissue can be metabolically obese, with insulin resistance and dyslipidemia. ${ }^{7,8}$

In this Lagos study, in both gender, it is not all that have high abdominal adiposity that have hyperlipidemia and it is not all that have hyperlipidemia that have high abdominal adiposity. In male, minority number of men with high abdominal adiposity has hyperlipidemia $(16.67 \%)$ while sizable minority of women with high abdominal adiposity had hyperlipidemia $(28.21 \%)$. However the disparity lies in the fact that though both gender have minority number of subjects with high abdominal adiposity with hyperlipidaemia, the male gender have fewer minority number $(16.67 \%$, approximately 1 in every 6 male) than female with sizable minority $(28.21 \%$, approximately 1 in 3 or 4 female).

A marked disparity was noticed between gender with respect to those with hyperlipidemia and high abdominal adiposity. In male subjects with hyperlipidemia the subject with high abdominal adiposity is in the minority (25\%) while in the female subjects with hyperlipidemia, those with high abdominal adiposity are in the majority $(78.57 \%)$. The disparity here is marked and of significant prognostic importance.

In a study of adolescent in Canada, it was found that the metabolic syndrome was completely absent in adolescent with low intrabdominal fat and was present in $13.8 \%$ of males and $8.3 \%$ of females with high intrabdominal fat on magnetic resonance imaging. ${ }^{9}$

In post menopausal women, the associations of abdominal adiposity, fasting serum levels of insulin, and sex hormones with blood lipids, lipoproteins, and apolipoproteins A-I and B were studied cross- 
sectionally in 75 healthy, postmenopausal white women. In univariate analyses, abdominal adiposity (increased waist-to-hip girth ratio) and fasting insulin concentrations were negatively and significantly associated $(\mathrm{P}<0.05)$ with plasma high density lipoprotein cholesterol $(\mathrm{r}=-0.47$ and -0.38 , respectively) and apolipoprotein A-I $(\mathrm{r}=-0.37$ and -0.36$)$, and positively associated with $\log$ triglycerides $(\mathrm{r}=0.54$ and 0.33$)$ and apolipoprotein $B(r=0.43$ and 0.22$)$. Sex hormone binding globulin was positively and significantly associated with high density lipoprotein cholesterol $(\mathrm{r}=0.32)$ and negatively associated with $\log$ triglyceride $(r=-0.45)$ and apolipoprotein $B(r=-0.36) .{ }^{10}$

In post menopausal black women in this study, hyperlipidemia was found in majority in their 7th decade of life suggesting that post menopausal black women are more likely to have hyperlipidemia few years after attainment of the post menopausal status (this is an hypothesis for future study in post-menopausal black women) while none (no hyperlipidemia) was recorded in the eighth and ninth decade. To conclude that there is no hyperlipidemia in black women in this age group may be more of a fallacy than reality, a more acceptable explanation is that either those with hyperlipidemia in eighth and ninth decades of life were not represented in this sample during random sampling or they have become victim of mortality due to the many causes of mortality in the elderly.

\section{Conclusion}

In both gender minority number of those with high abdominal adiposity have hyperlipidaemia though with female having sizable minority number.

Conversely a marked disparity is seen among female subjects with hyperlipidemia as majority (78.57\%) had high abdominal adiposity while among male with hyperlipidemia only minority $(25 \%)$ had high abdominal adiposity. The disparity here is marked and of significant cardiovascular prognostic importance in Lagos southwest Nigeria.

\section{Acknowledgments}

None.

\section{Conflict of interest}

The author declares there is no conflict of interest.

\section{References}

1. Wang J, Heymsfield SB, Aulet M, et al. Body fat from body density: underwater weighing vs. dual-photon absorptiometry. Am J Physiol. 1989;256(6 Pt 1):E829-34.

2. Wang Y, Rimm EB, Stampfer MJ, et al. Comparison of abdominal adiposity and overall obesity in predicting risk of type 2 diabetes among men. Am J Clin Nutr. 2005;81(3):555-563.

3. Carr MC, Brunzell JD. Abdominal Obesity and Dyslipidemia in the Metabolic Syndrome: Importance of Type 2 Diabetes and Familial Combined Hyperlipidemia in Coronary Artery Disease Risk. J Clin Endocrinol Metab. 2004;89(6):2601-7.

4. McNamara JR, Campos H Ordovas J, et al. Effect of gender, age, and lipid status on low density lipoprotein subfraction distribution. Results of the Framingham Offspring Study. Arteriosclerosis. 1987;7(5):483-490.

5. Swinkels D, Demacker P, Hendriks J, et al. Low density lipoprotein subfractions and relationship to other risk factors for coronary artery disease in healthy individuals. Arteriosclerosis. 1989;9(5):604-13.

6. Austin MA, King MC, Vranizan KM, et al. Atherogenic lipoprotein phenotype. A proposed genetic marker for coronary heart disease risk. Circulation. 1990;82:495-506.

7. Fujimoto W, Abbate $\mathrm{S}$, Kahn S, et al. The visceral adiposity syndrome in Japanese-American men. Obes Res. 2(4):364-371.

8. Ruderman N, Chisholm D, Pi-Sunyer X, et al. The metabolically obese, normal-weight individual revisited. Diabetes. 47(5):699-713.

9. Syme C, Abrahamowicz M, Leonard GT, et al. Intra-Abdominal Adiposity and individual Components of the Metabolic Syndrome in Adolescent Sex difference and Underlying Mechanism. Arc Paediatr Adolesc Med. 2008;162(5):453-461.

10. Soler JT, Folsom AR, Kaye SA, et al. Associations of abdominal adiposity, fasting insulin, sex hormone binding globulin, and estrone with lipids and lipoproteins in post-menopausal women. Atherosclerosis. $1989 ; 79(1): 21-7$ 